\title{
The Role of Micro-RNAs in Post-Traumatic Stress Disorder
}

\author{
Ali Shahini ${ }^{1}$, Zabihollah Khaksar ${ }^{2}$, Sajad Sahab Negah ${ }^{1,3 *}$ \\ ${ }^{1}$ Department of Neuroscience, Mashhad University of Medical Sciences, Mashhad, Iran \\ ${ }^{2}$ Department of Basic Sciences, School of Veterinary Medicine, Shiraz University, Shiraz, Iran \\ ${ }^{3}$ Shefa Neuroscience Research Center, Khatam Alanbia Hospital, Tehran, Iran
}

\section{A BSTRACT}

Introduction: Post-traumatic stress disorder (PTSD) is a form of anxiety disorders. PTSD typically follows an exposure to severe stress. It is characterized by a particular clinical syndrome, including re-experiencing symptoms and alterations in arousal, avoidance, cognition, and mood conditions. Recent evidences propose that micro-RNAs are crucial epigenetic players in mental disorders. It has been demonstrated that micro-RNAs are promising non-invasive biomarkers for different mental health disorders. It has been reported that the levels of micro-RNAs have been changed in psychotic diseases, including Alzheimer's disease and PTSD. Conclusion: Micro-RNAs seem to play a role in pathophysiology of psychological diseases. In this article, we discussed the new findings suggesting a relationship between micro-RNAs and PTSD as well as the possible roles of micro-RNAs in diagnosis and treatment of this mental disorder.

*Corresponding Author: Sajad Sahab Negah

E-mail: sahabnegahs@mums.ac.ir 
نقش micro-RNA ها در اختلال اضطرابى گيس از ضربه
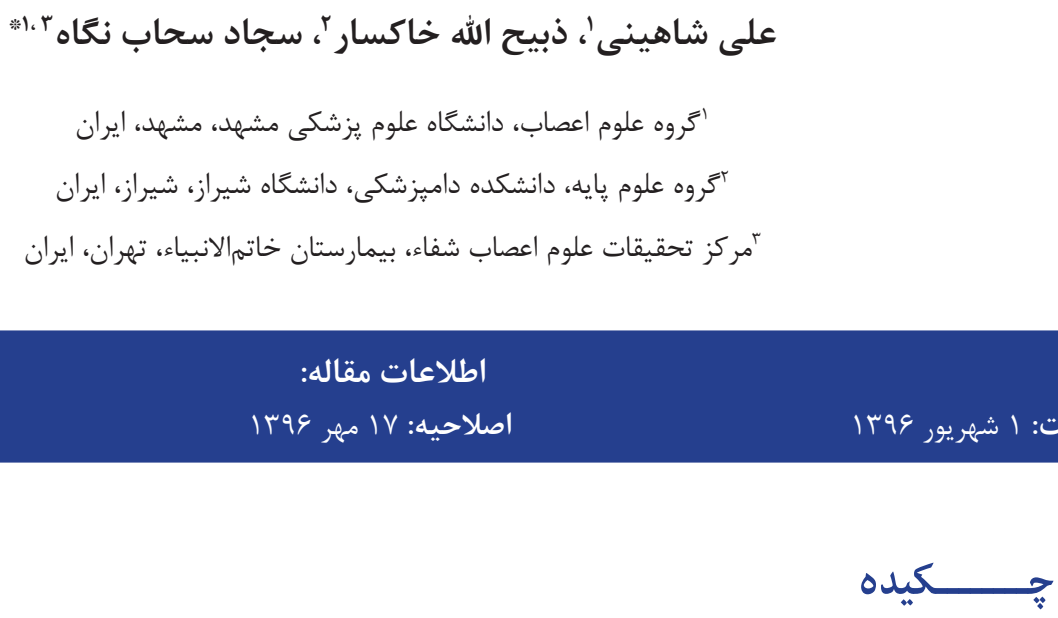

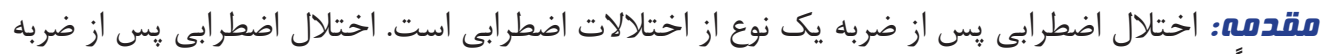

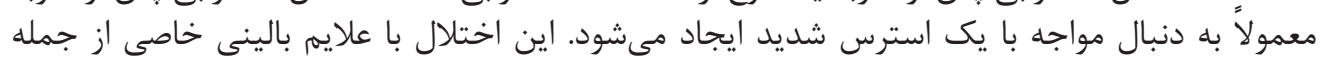

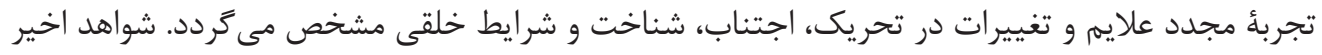

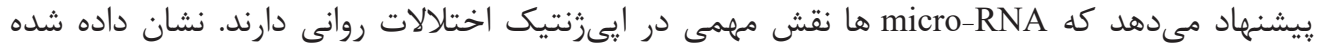

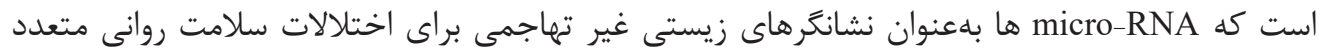

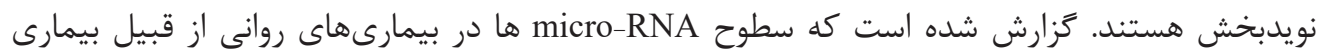

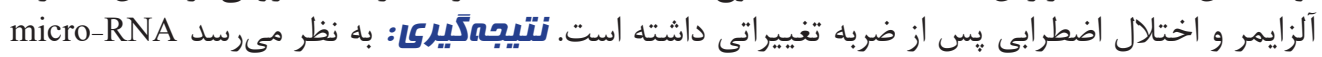

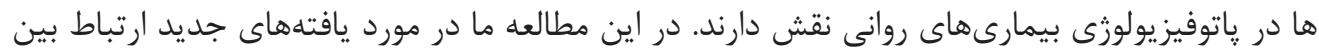
micro-RNA

$$
\text { و درمان اختلالات روانى بحث مى كنيه. }
$$

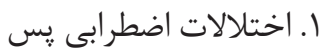

ازب

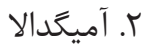

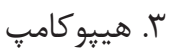

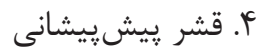

" نويسنده مسئول: سجاد سحاب نعاه آدرس الكترونيكى: sahabnegahs@mums.ac.ir 


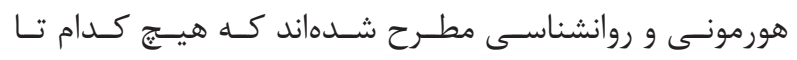

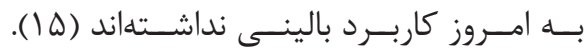

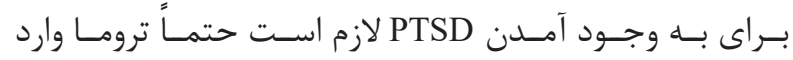

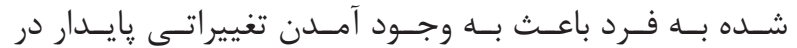

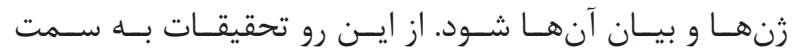

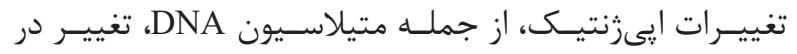

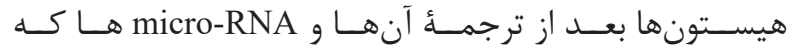

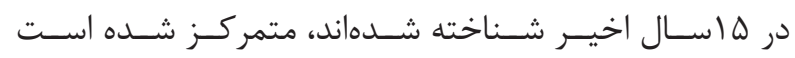

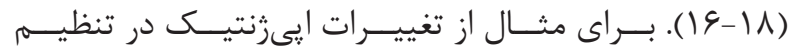

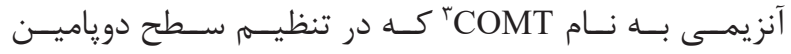

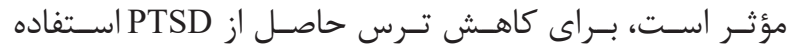

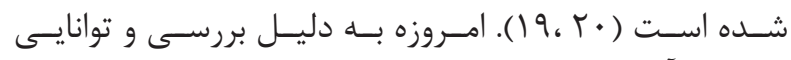

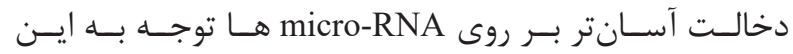

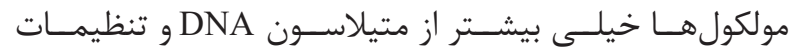

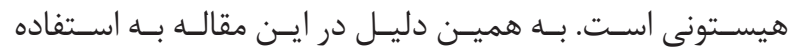

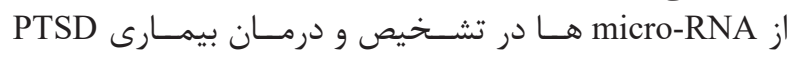

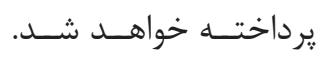

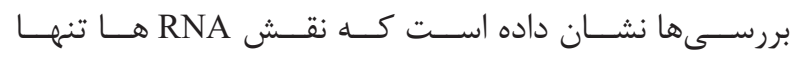

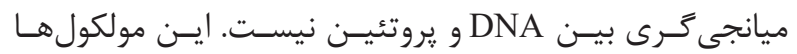

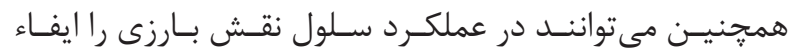

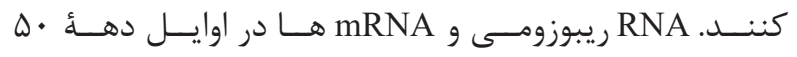

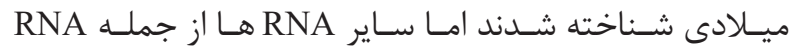

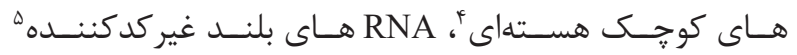

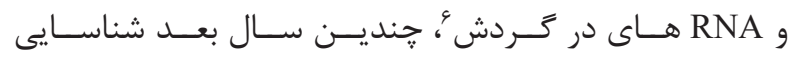

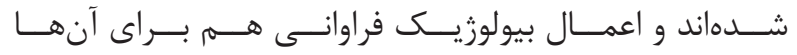

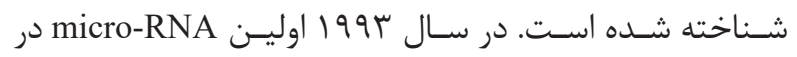

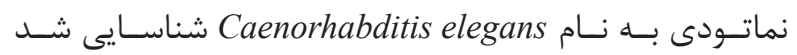

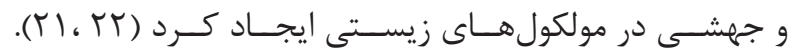

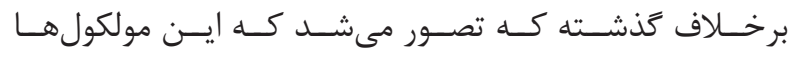

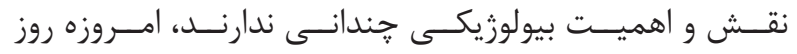

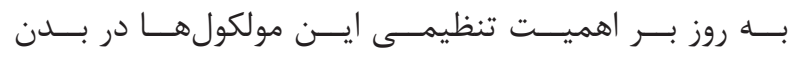

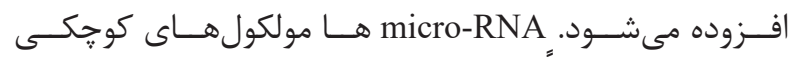

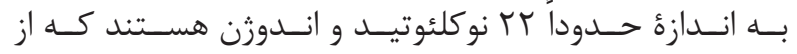

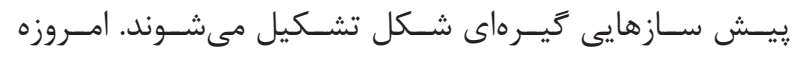

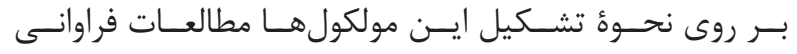

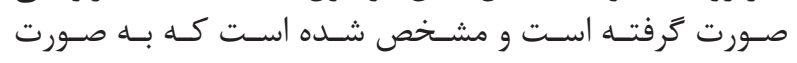

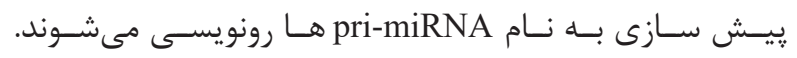

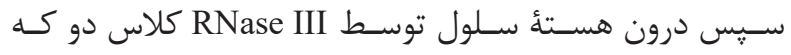

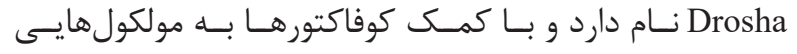

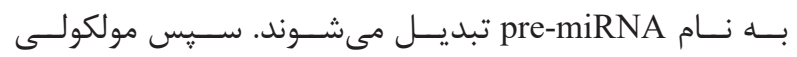
بــــ نـام

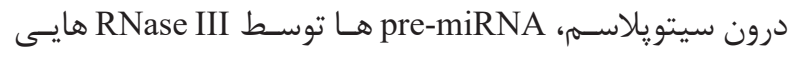

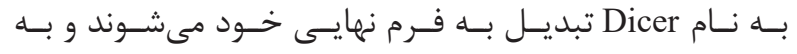

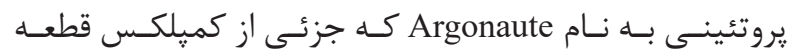

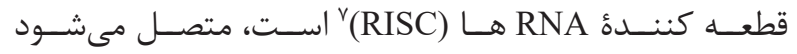

${ }^{1}$ Post-traumatic stress disorder

${ }^{2}$ Magnetic resonance imaging

${ }^{3}$ Catechol-O-methyl transferase

${ }^{4}$ Small nuclear RNA

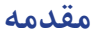

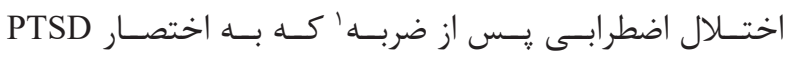

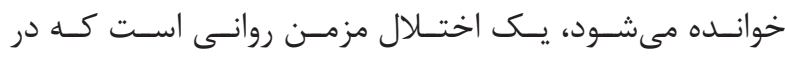

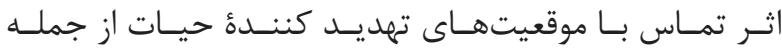

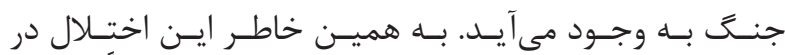

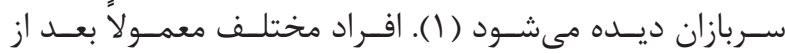

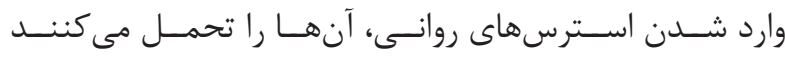

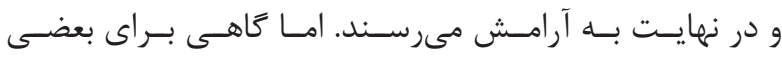

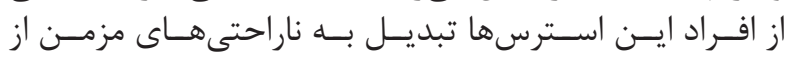

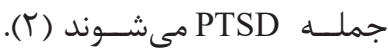

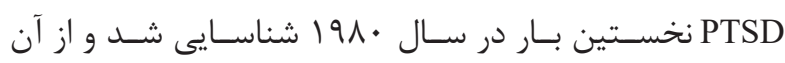

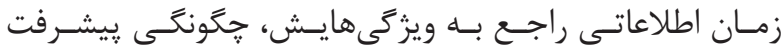

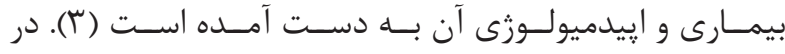

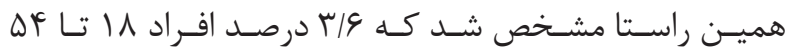

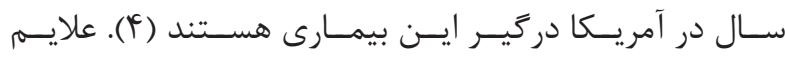

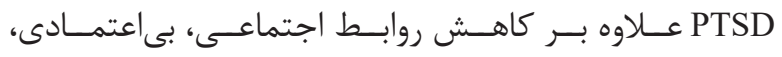

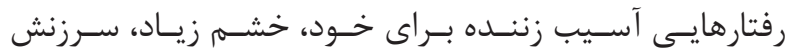

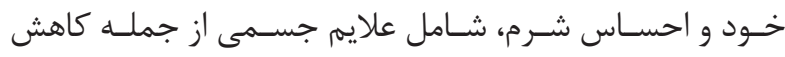

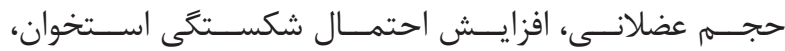

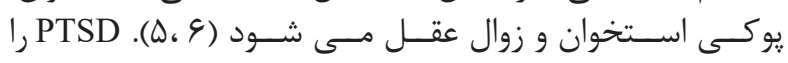

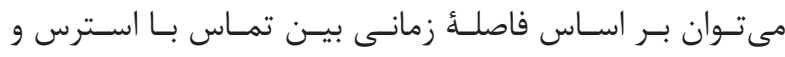

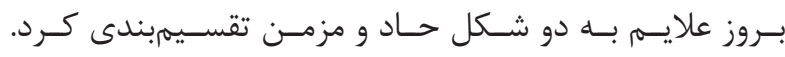

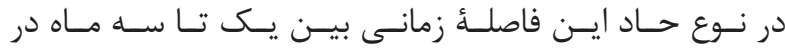

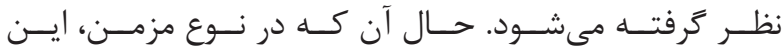

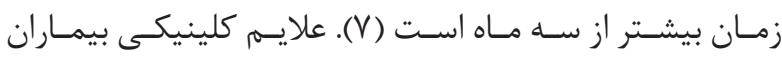

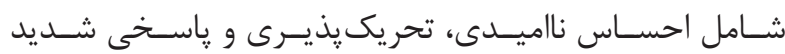

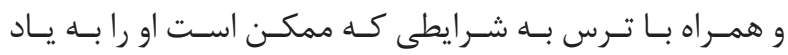

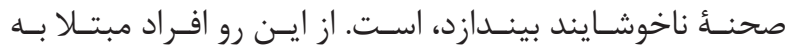

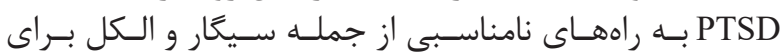

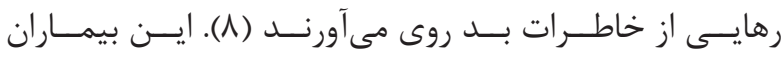

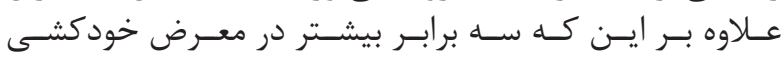

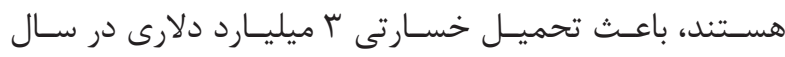

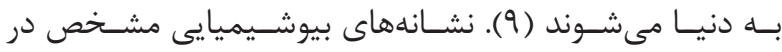

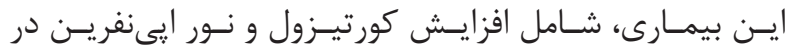

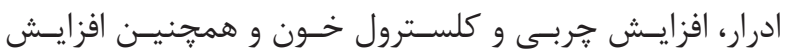

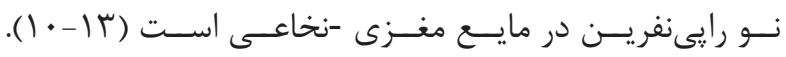

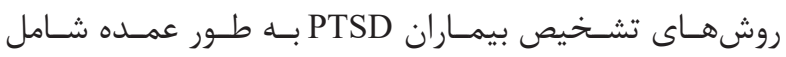

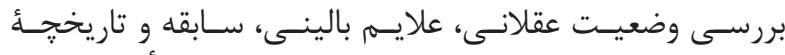

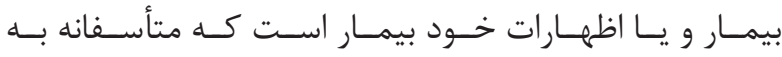

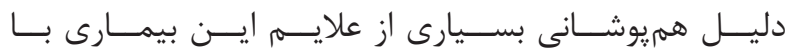

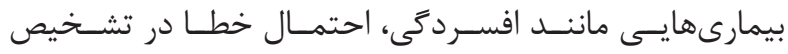

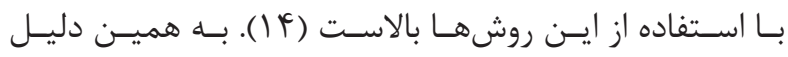

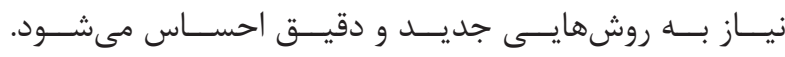

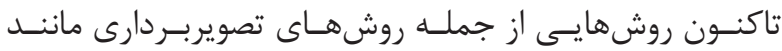

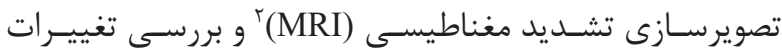

${ }^{5}$ Long non-coding RNA

${ }^{6}$ Circulatory RNA

${ }^{7}$ RNA-Induced slicing complex 


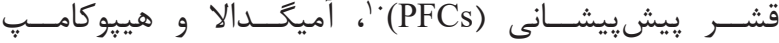

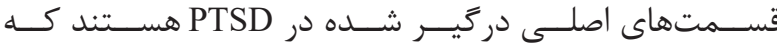

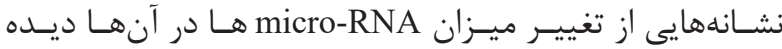

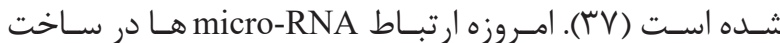

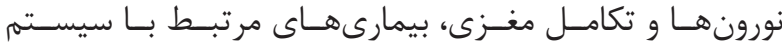

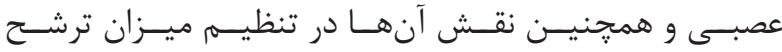

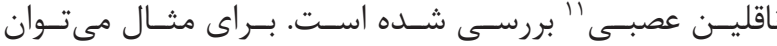

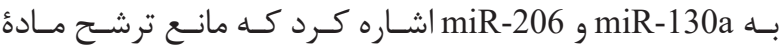

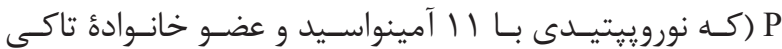

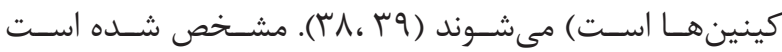

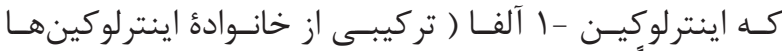

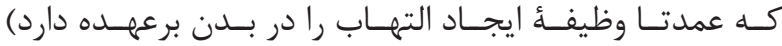

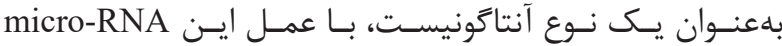

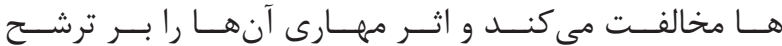

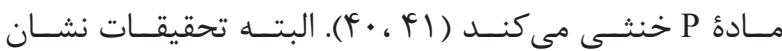

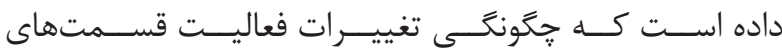

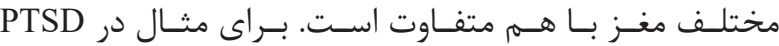

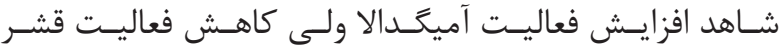

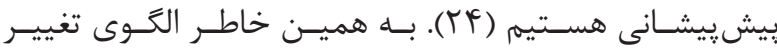

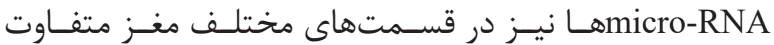

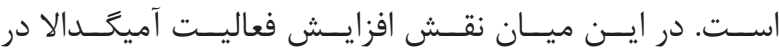

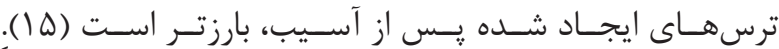

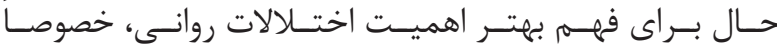

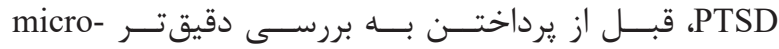

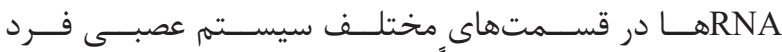

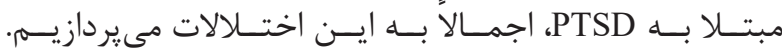

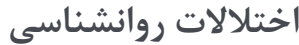

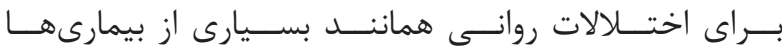

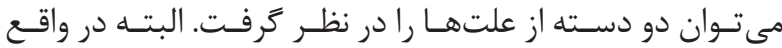

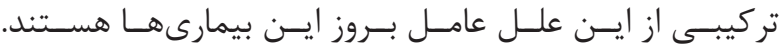

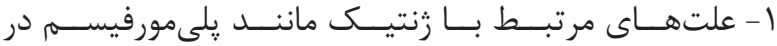

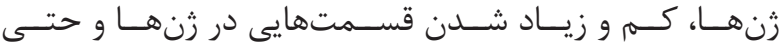

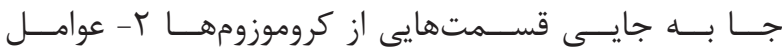

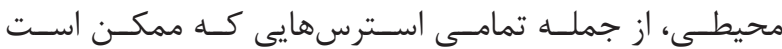

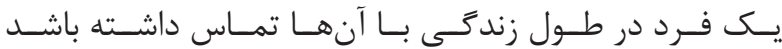

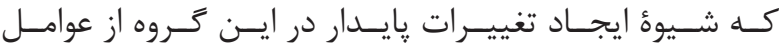

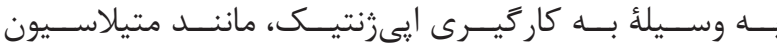

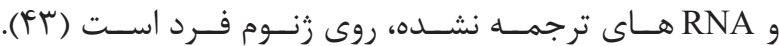

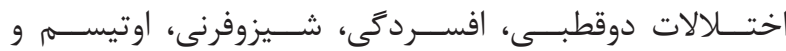

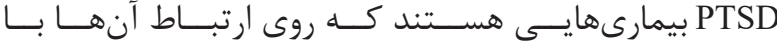

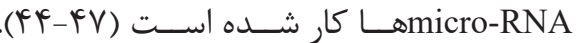

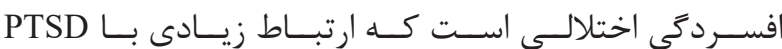

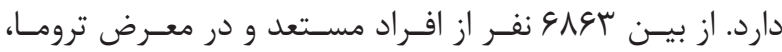

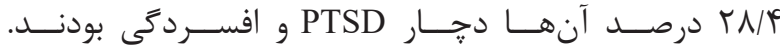

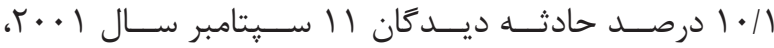

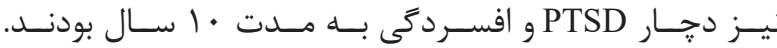

\footnotetext{
${ }^{8}$ Biomarker

${ }^{9}$ Mild cognitive impairment
}

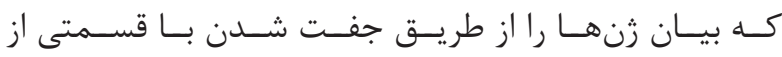

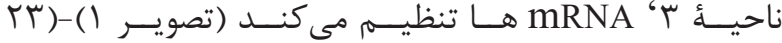

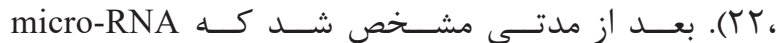

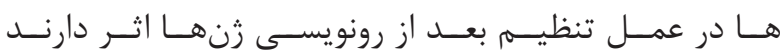

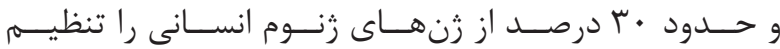

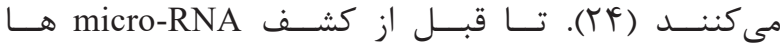

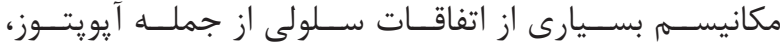

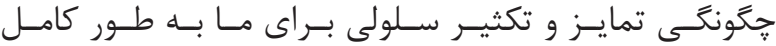

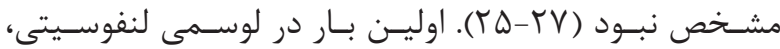

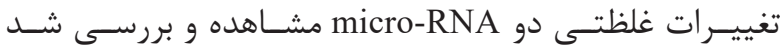

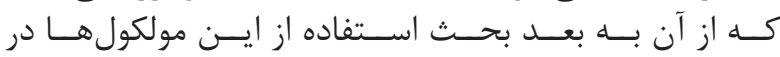

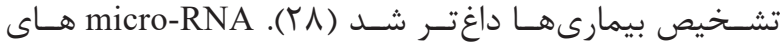

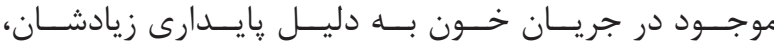

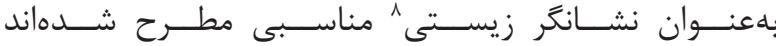

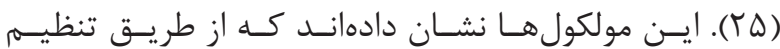

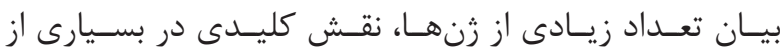

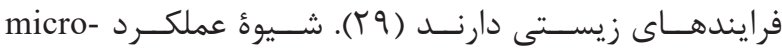

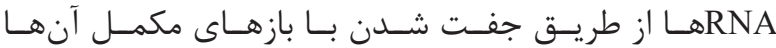

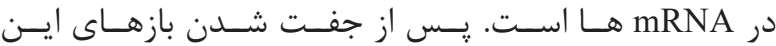

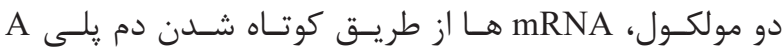

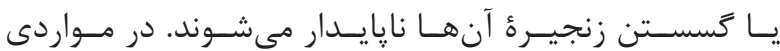

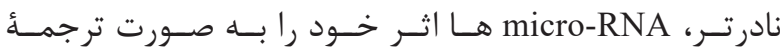

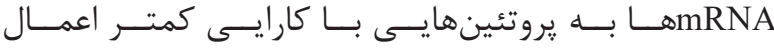

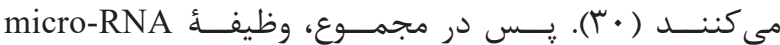

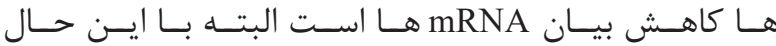

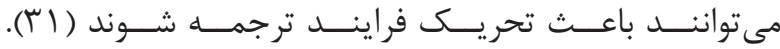

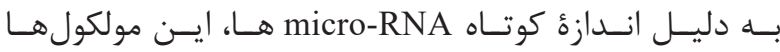

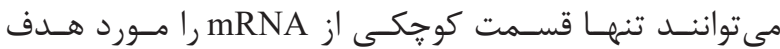

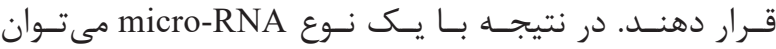

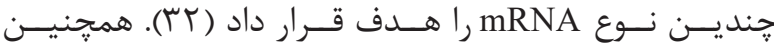

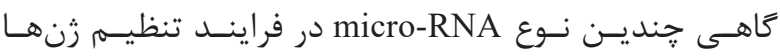

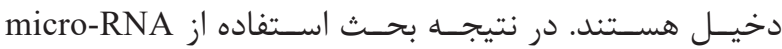

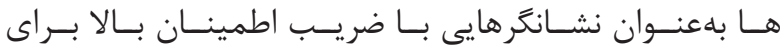

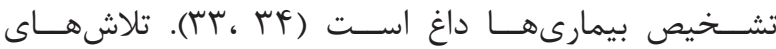

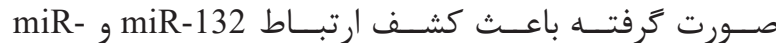

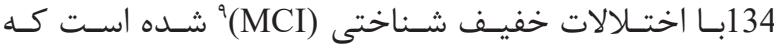

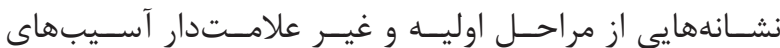

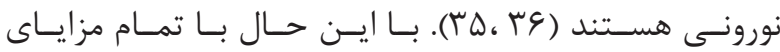

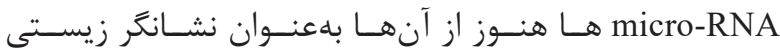

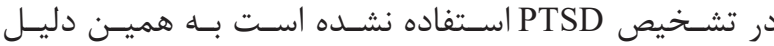

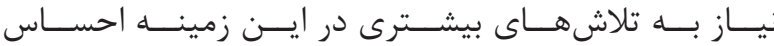

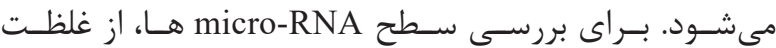

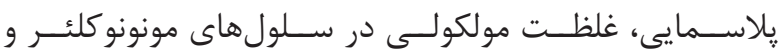

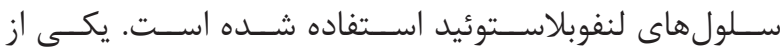

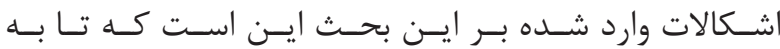

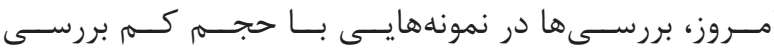

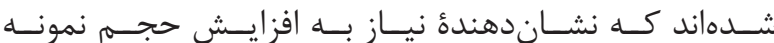

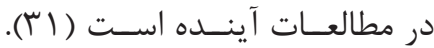

${ }^{10}$ Prefrontal cortex

${ }^{11}$ Neurotransmitters 


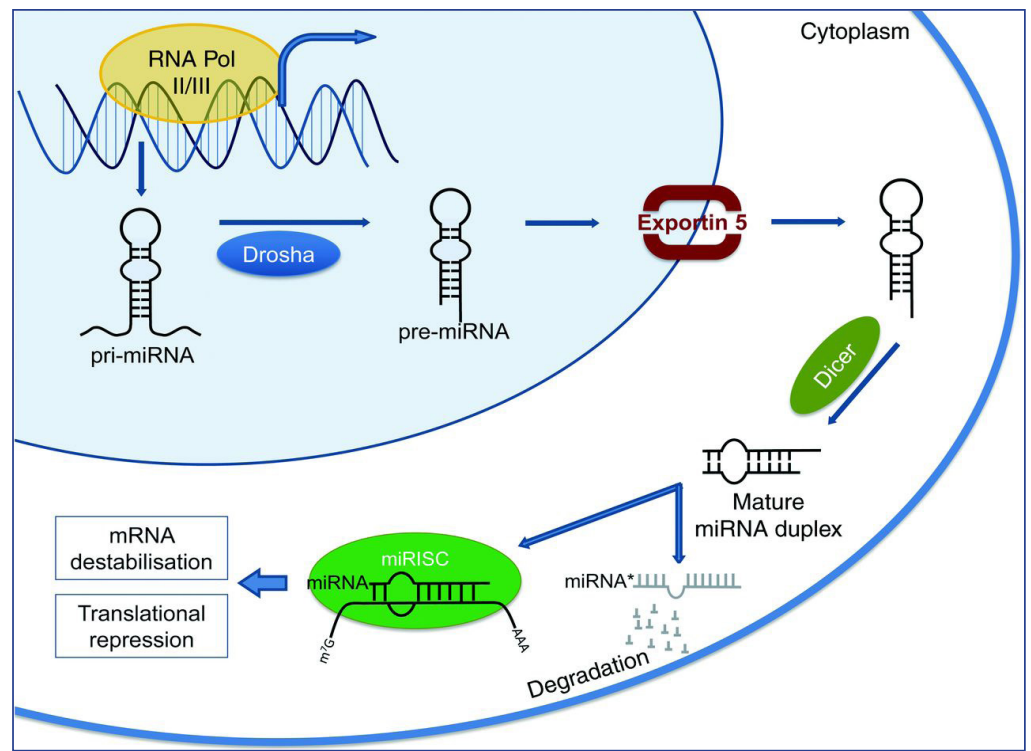

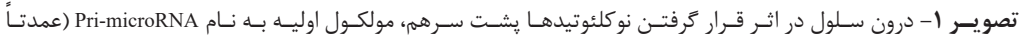

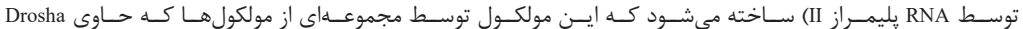

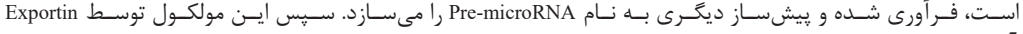

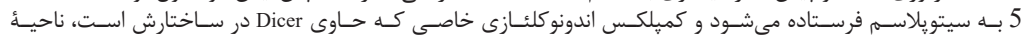

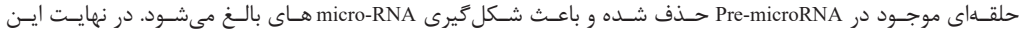

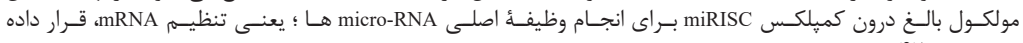

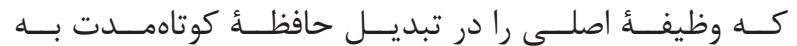

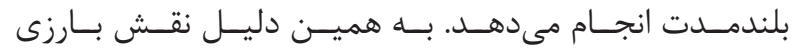

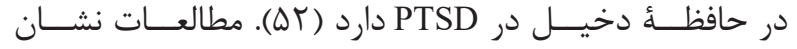

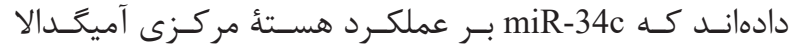

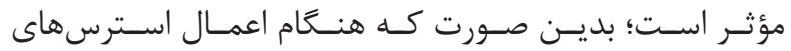

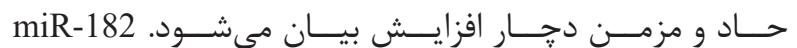

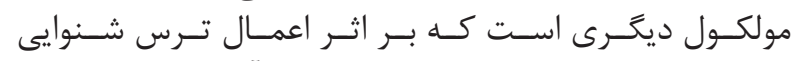

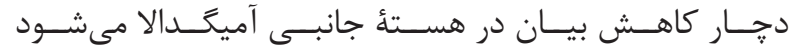

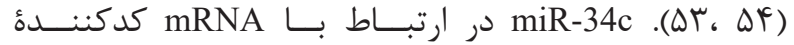

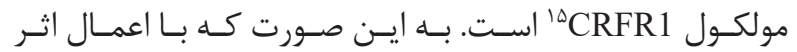

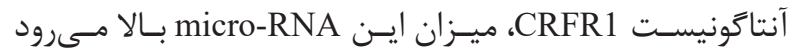

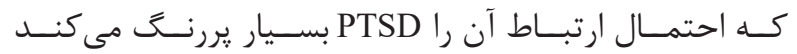

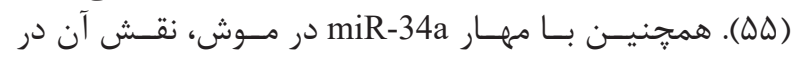

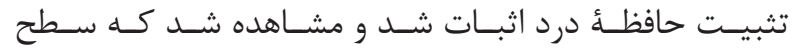

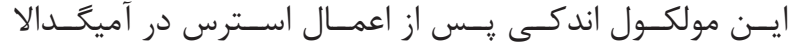

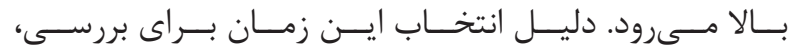

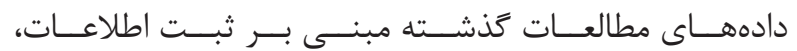

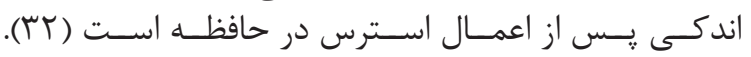

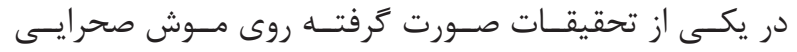

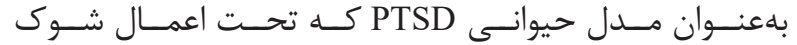

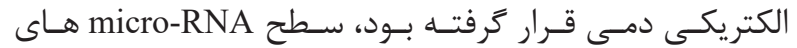

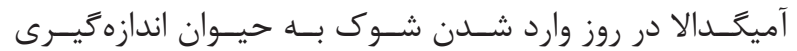

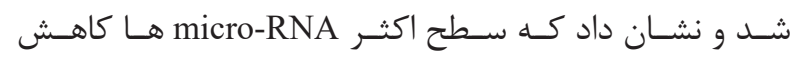

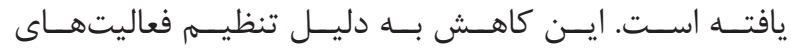

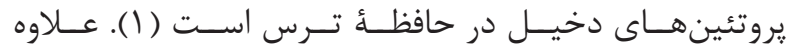

\section{${ }^{12}$ Pathogenicity}

${ }^{13} \mathrm{~K} 506$ binding protein 5

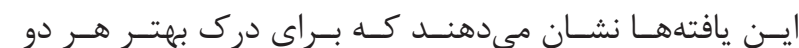

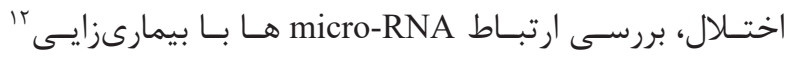

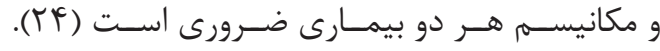

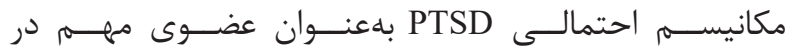

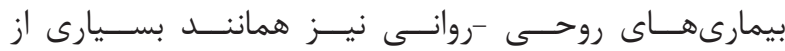

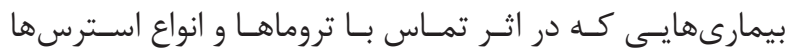

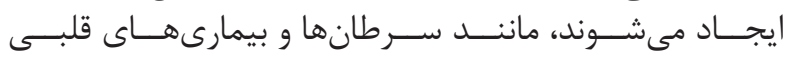

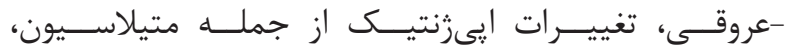

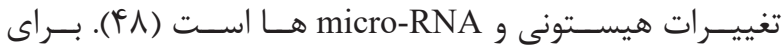

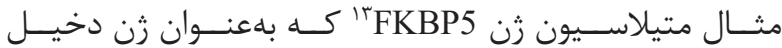

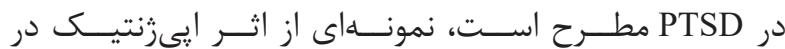

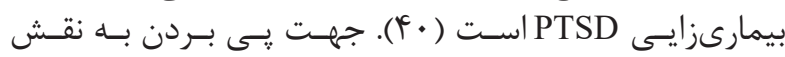

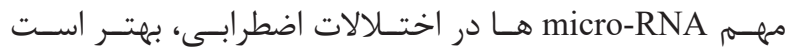

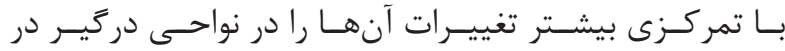

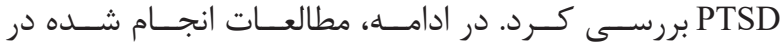

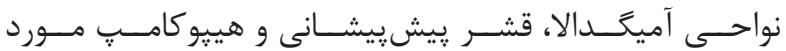

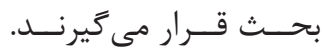

ارتباط آميعَدالا و micro-RNA ها در فرد دجار PTSD آميخــدالا ناحيـهاى بادامسى شـكل در مغـز اسـت. ناحيههايـى

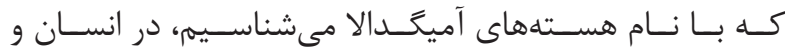

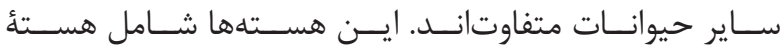

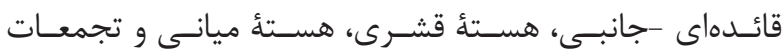

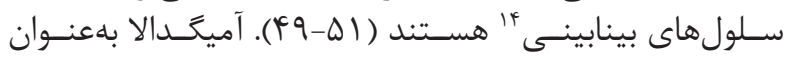

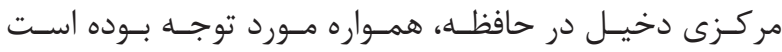

${ }^{14}$ Intermediate cells (ITCs)

${ }^{15}$ Corticotrophin releasing factor receptor 


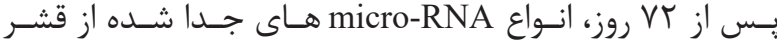

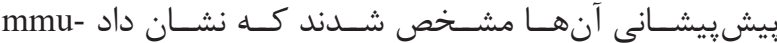
و mmu-miR-19473p ،ebv-miR-BART8-3p ،miR-1971

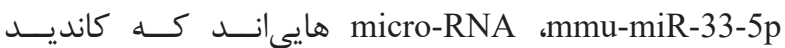

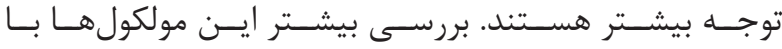

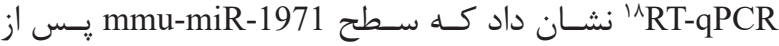

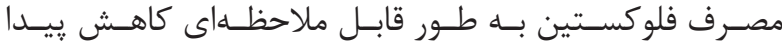

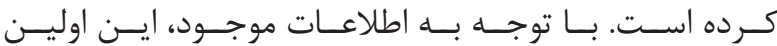

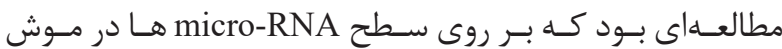

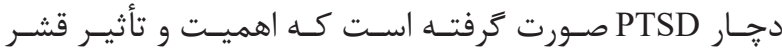

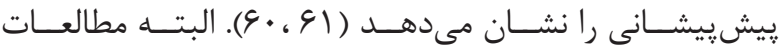

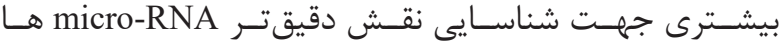

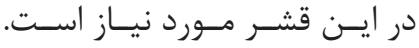

ارتباط هيبو كامب و micro-RNA ها در فرد دجار PTSD

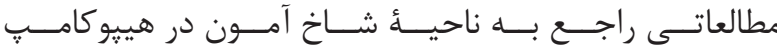

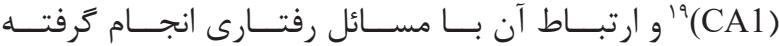

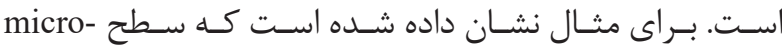

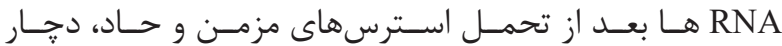

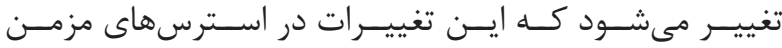

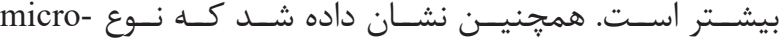

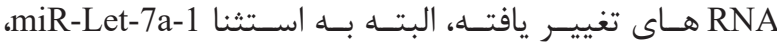

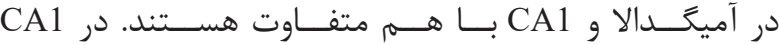
تغيــرات مشـاهده شـده شـامل افزايسش

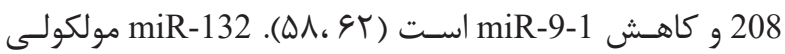

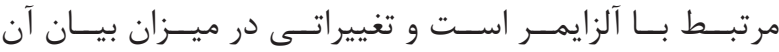

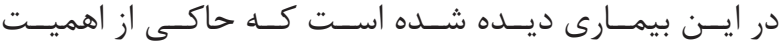

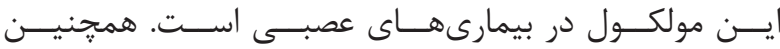

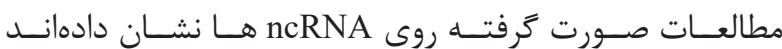

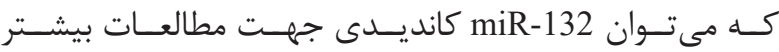

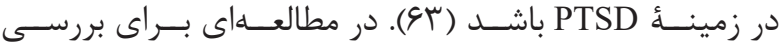

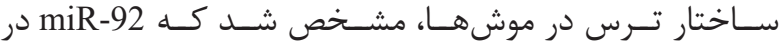

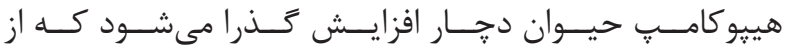

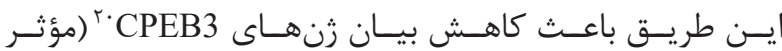

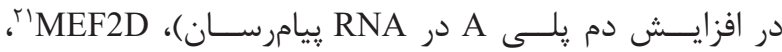
KCC2

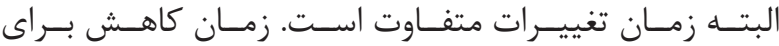

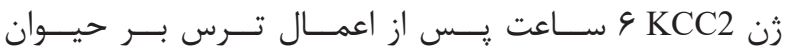

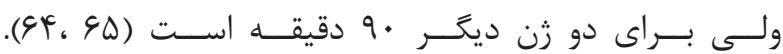

نقش micro-RNA ها در درمان PTSD

امــروزه بــراى درمــان PTSD روى علايـهم مختلـف آن ماننــد

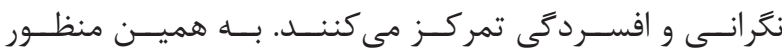

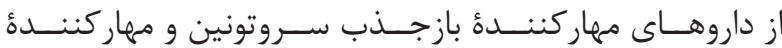

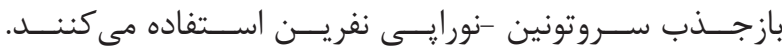

${ }^{16}$ Mus musculus miR

${ }^{17}$ Fluoxetine

${ }^{18}$ Quantitative reverse transcription PCR

${ }^{19}$ Cornu ammonis

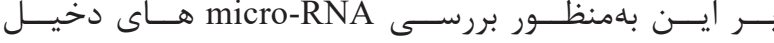

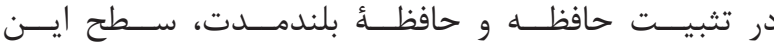

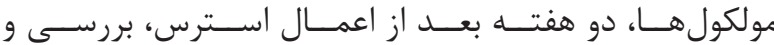

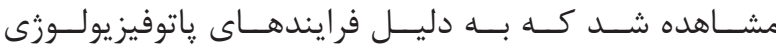

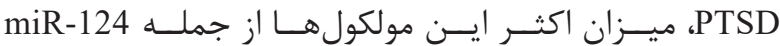

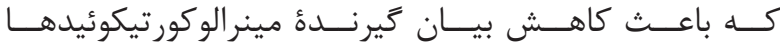

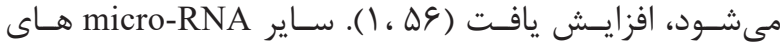

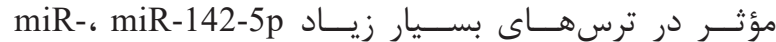

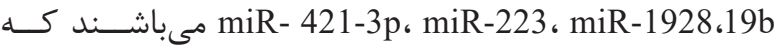

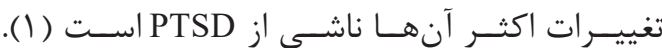

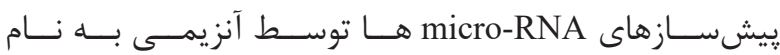

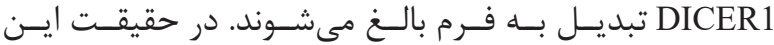

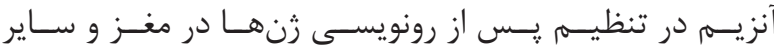

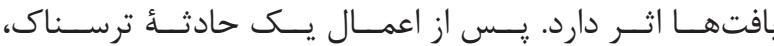

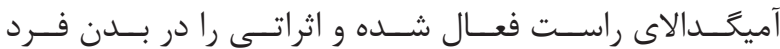

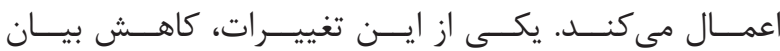

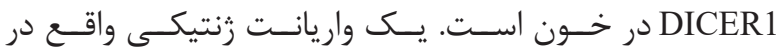

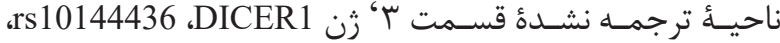

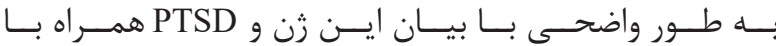

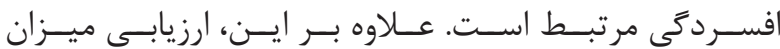

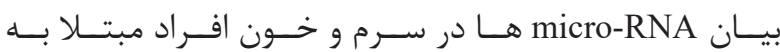

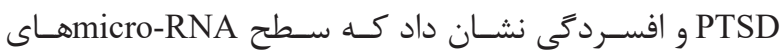

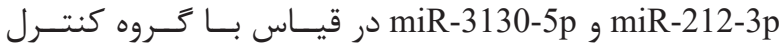

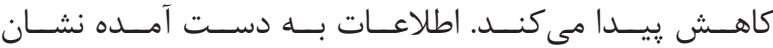

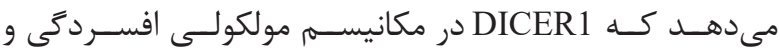

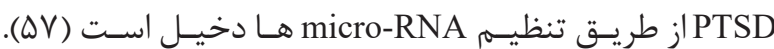

ارتباط قشر ييش

PTSD

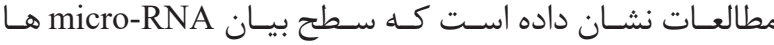

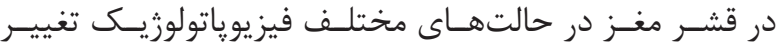

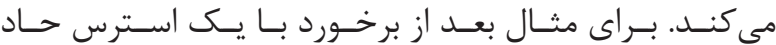

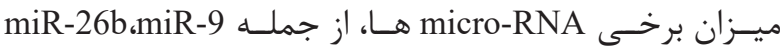
مiR-494 miR-30b ،miR-29b ،

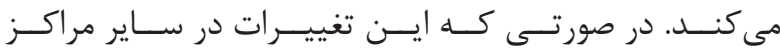

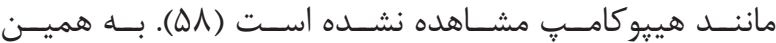

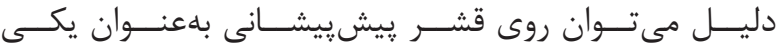

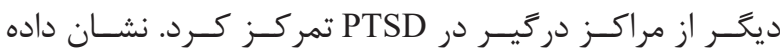

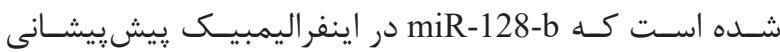

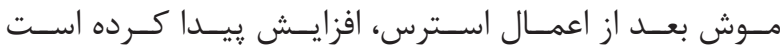

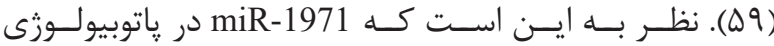

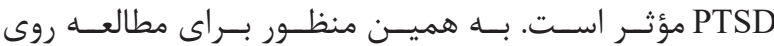

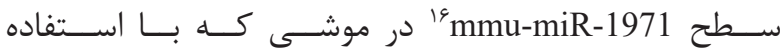

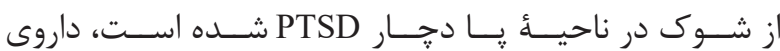

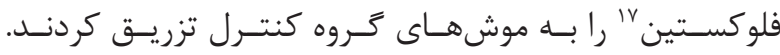

${ }^{20}$ Cytoplasmic polyadenylation element binding protein 3

${ }^{21}$ Myocyte-specific enhancer factor 2D

${ }^{22}$ Is also called potassium-chloride transporter member 5 


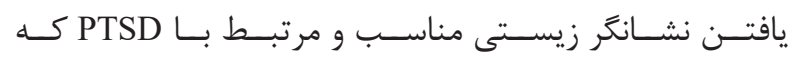

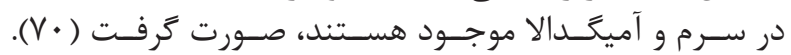

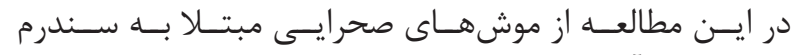

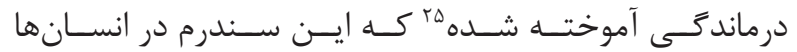

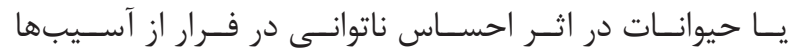

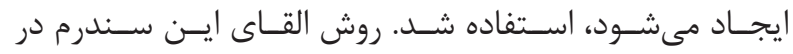

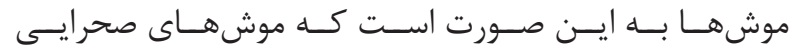

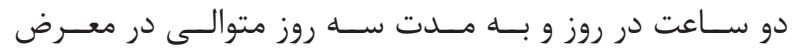

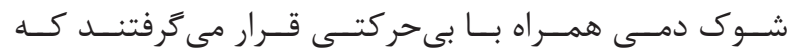

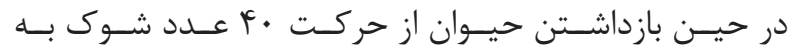

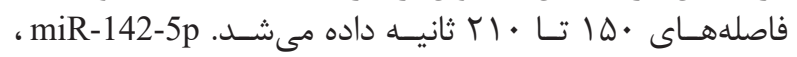
miR-324،miR-322،miR-223-3p ، miR-1928 ،miR-19b miR-463،miR-421-3p ،

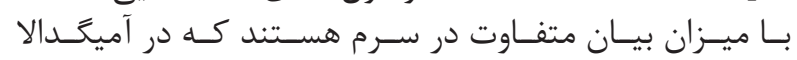

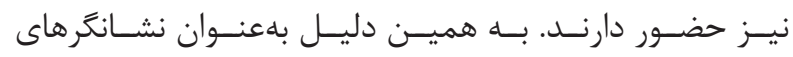

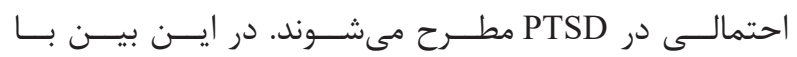

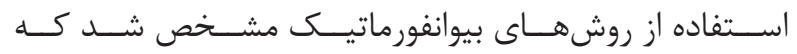

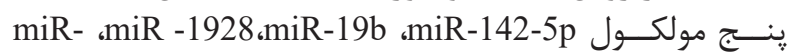

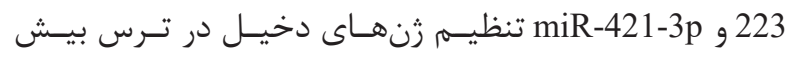

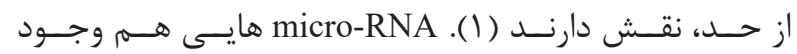

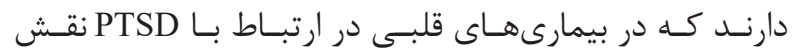

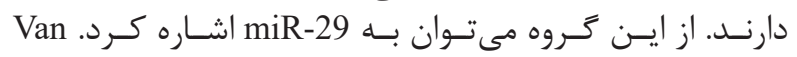

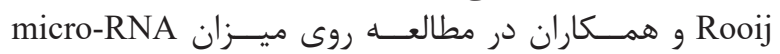

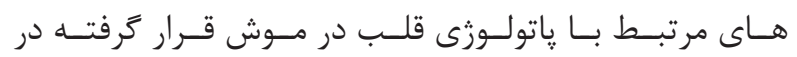

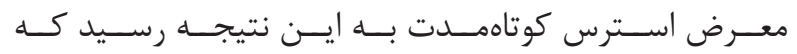

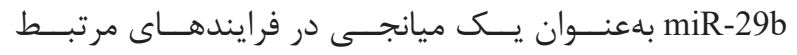

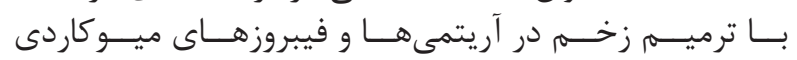

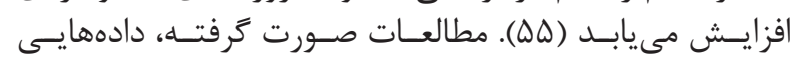

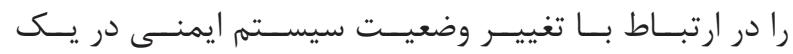

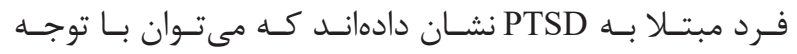

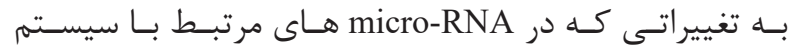

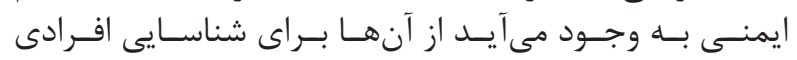

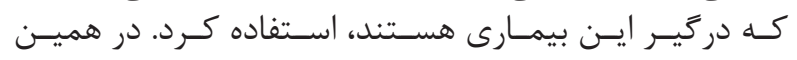

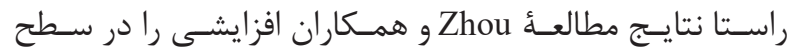

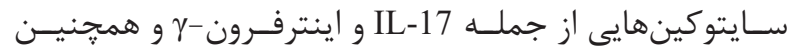

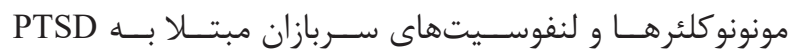

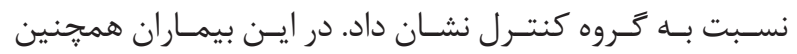

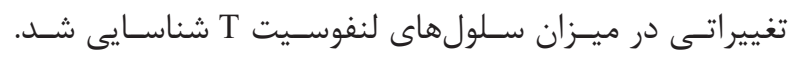

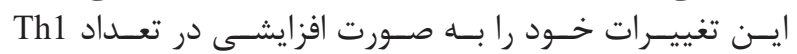

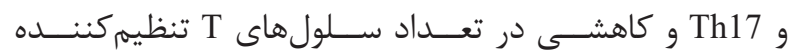

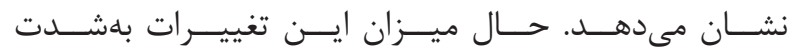

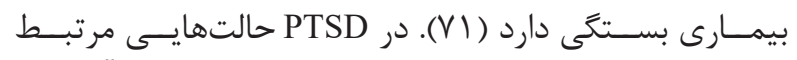

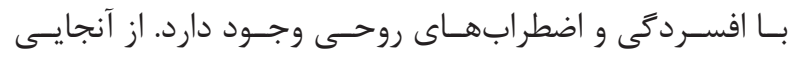

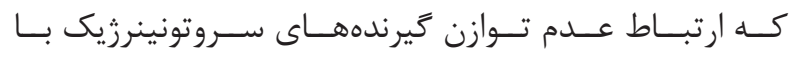

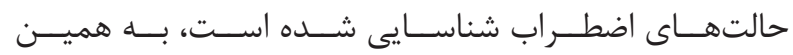

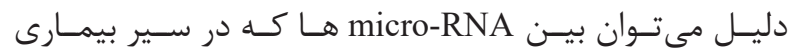

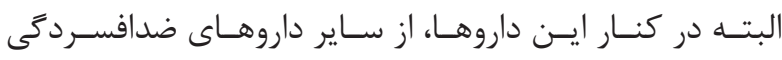

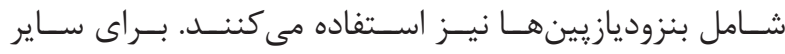

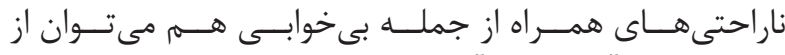

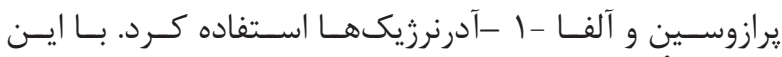

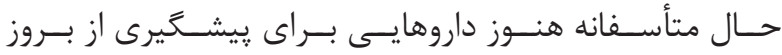

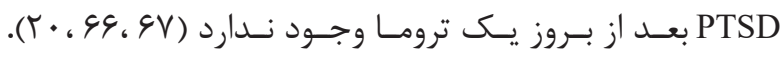

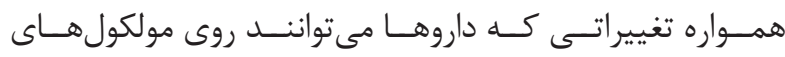

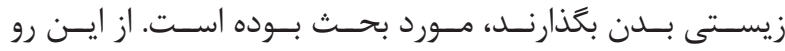

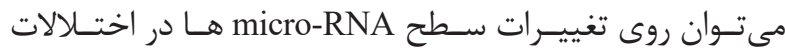

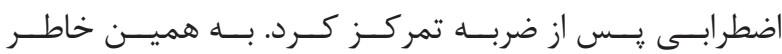

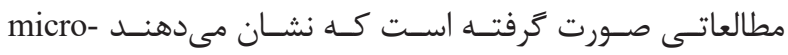

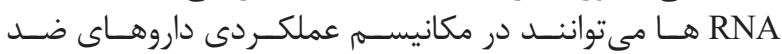
افسـرد

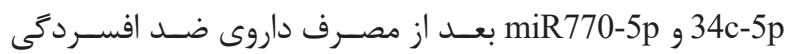
Paroxetine Sو Escitalopram

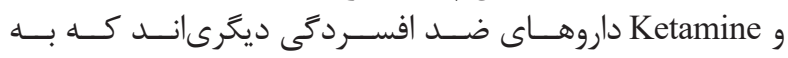

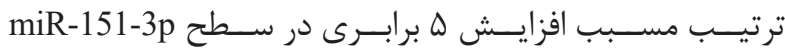

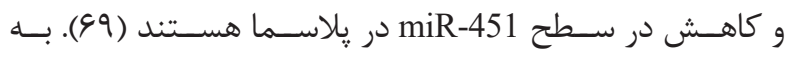

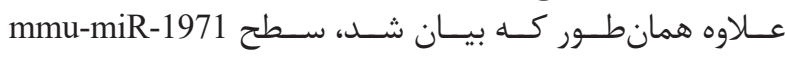

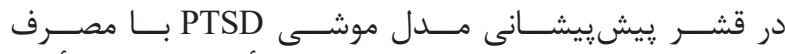

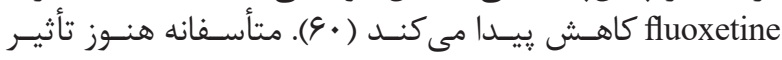

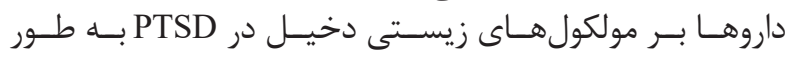

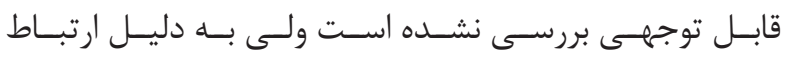

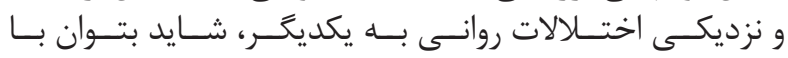

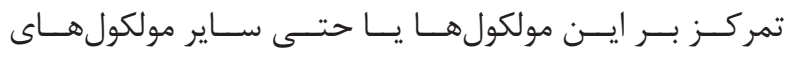

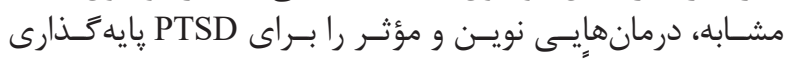

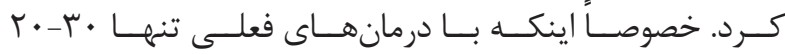

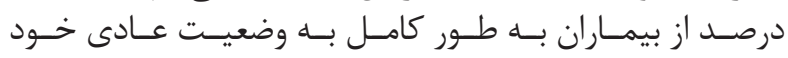

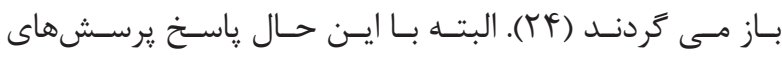

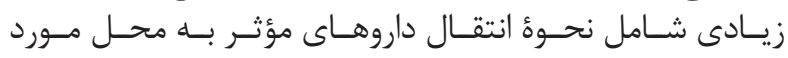

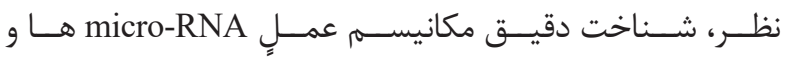

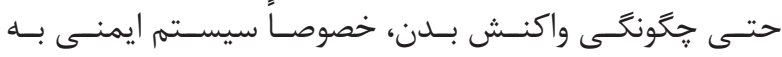

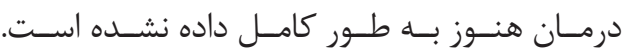

نقش micro-RNA ها در تشخيص PTSD

micro-RNA

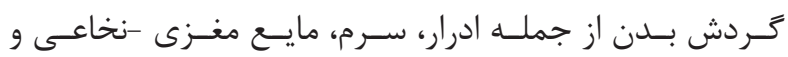

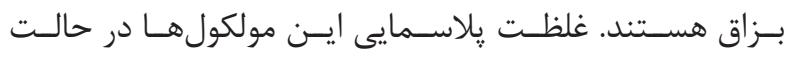

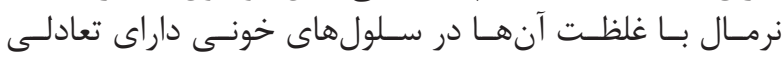

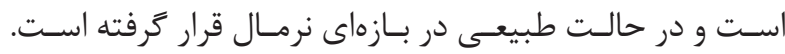

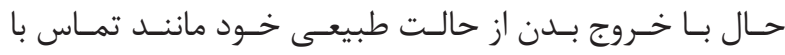

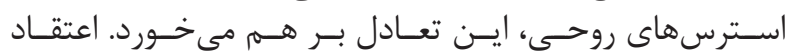

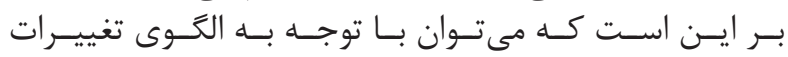

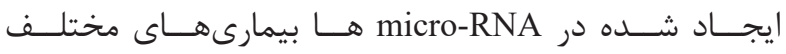

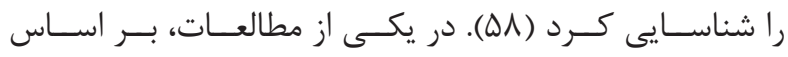

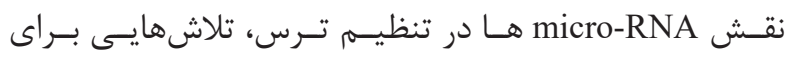

\footnotetext{
${ }^{23}$ Anti-depressant

${ }^{24}$ The brand name is Lexapro

${ }^{25}$ Helplessness syndrome
} 


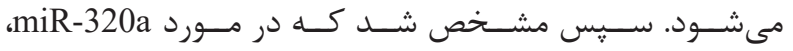

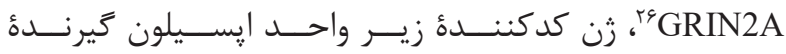

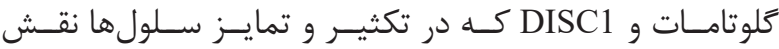

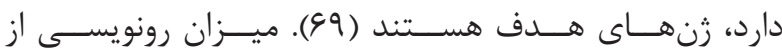

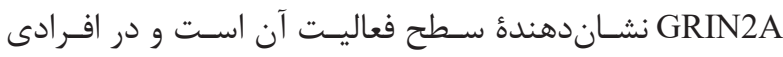

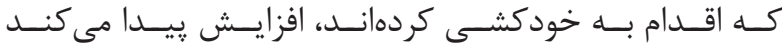
(VT)

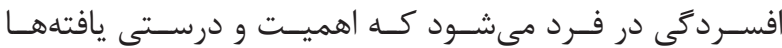

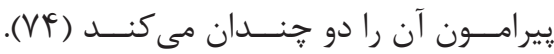

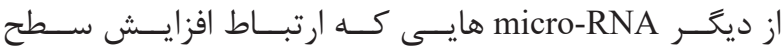

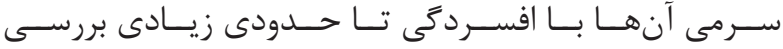

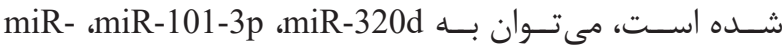
miR-423-5p ، و اش miR-93-5p-5p

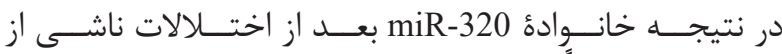

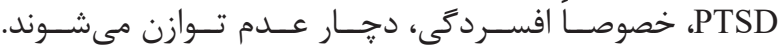

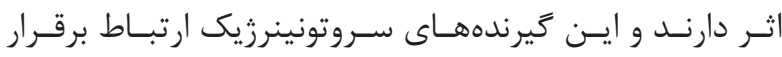

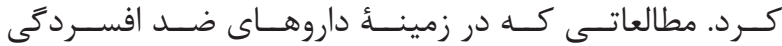

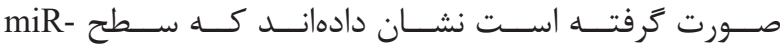

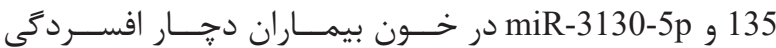

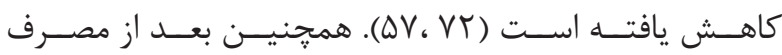

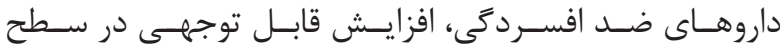

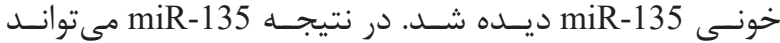

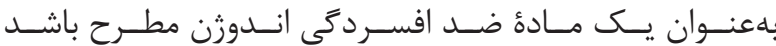

در مطالعـهاى ييرامـون سـطح micro-RNA هــا در يلاسـماى

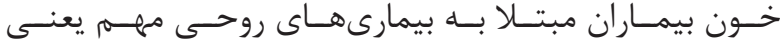

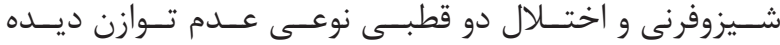

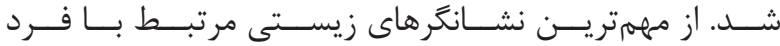

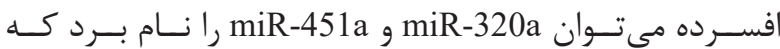

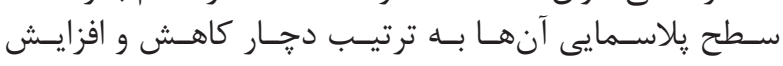

\begin{tabular}{|c|c|c|c|}
\hline شمارة منبع & محل مورد مطالعه & دليل اهميت & توع micro-RNA \\
\hline$(\Delta r \cdot \Delta F)$ & سنترال آميخدالا & نقش احتمالى در حافظه بعد از & miR-34c \\
\hline$(\Delta r, \Delta F)$ & لترال آميخددالا & نقش احتمالى در حافظه بعد از & miR-182 \\
\hline (rT) & آميكَدالا موش & تثبيت حافظةٔ درد & $\operatorname{miR}-34 a$ \\
\hline$(r \Delta)$ & سيستمم عصبى مركزى & نقش در اختلالات خفيف & miR-132 \\
\hline$(1, \Delta \varepsilon)$ & آميكدالا & مينر الوكورتيكونيدهيدا & miR-124 \\
\hline (1) & آميغدالا & نقش در ترس بسيار زياد ثاشىى & $\begin{array}{l}\text { miR-223.miR-1928 } \\
\text { miR-421-3p. }\end{array}$ \\
\hline$(\vee \&)$ & قشر مغز & نشاثلثرهاى زيستى احتمالى آسيب ناشى از & $\begin{array}{c}\text { miR-26b.miR-9 } \\
\text { miR-30b.miR-29b } \\
\text { miR-494. }\end{array}$ \\
\hline (४१) & 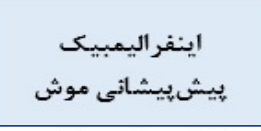 & 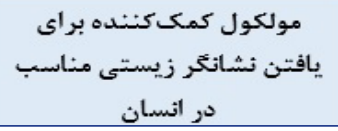 & miR-128-b \\
\hline$(\boldsymbol{4})$ & قشر يِيشييشانى & $\begin{array}{l}\text { كاهش يافته بعد از مصرف } \\
\text { fluoxetine }\end{array}$ & mmu-miR-1971 \\
\hline$(\varepsilon T, \vee q)$ & ناحيةُ شاخ آمون در & تغيير غلظت يس از اعمال & $\begin{array}{c}\text { miR-208 g miR-376b } \\
\text { miR-9-1 } g\end{array}$ \\
\hline$(\varphi f, \varphi \Delta)$ & هيبوكامب & $\begin{array}{c}\text { كاهش بيان زٔنهاى CPEB3 } \\
\text { KCC2.MEF2D }\end{array}$ & miR-92 \\
\hline$(9 \wedge)$ & 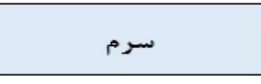 & $\begin{array}{c}\text { كاهش بعد از مصرف } \\
\text { escitalopram }\end{array}$ & $\begin{array}{l}9 \operatorname{miR}-34 c-5 p \\
\operatorname{miR} 770-5 p\end{array}$ \\
\hline (9१) & سرم ام & مصرف مايش ه برابرى بعد از & miR-151-3p \\
\hline (99) & 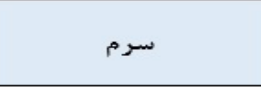 & كاهش غلظت يس از مصرف & $\operatorname{miR}-451$ \\
\hline (99) & 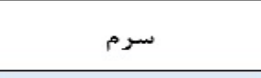 & $\begin{array}{c}\text { اثر روى زنهاى GRIN2A } \\
\text { DISC1 }\end{array}$ & miR-320a \\
\hline (VT) & سرم و مغز & مطرح شده بهعنوان مادة ضد افدرى اندورن & miR-135 \\
\hline 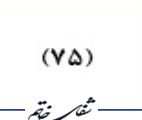 & 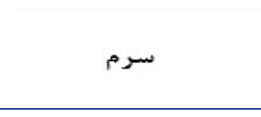 & داراى سطح سرمى مرتبط با & $\begin{array}{l}\text { miR-101-.miR-320d } \\
\text { miR- -miR-106-5p .3p } \\
\text { miR-93-5p , 423-5p }\end{array}$ \\
\hline
\end{tabular}

${ }^{26}$ Glutamate ionotropic receptor NMDA type subunit 2A 
مناسـب و امـكان اندازهخيـرى در قسـمتهاى مختلـف بـــن

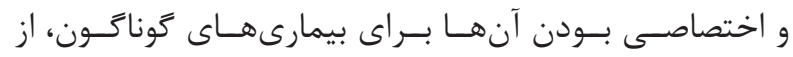

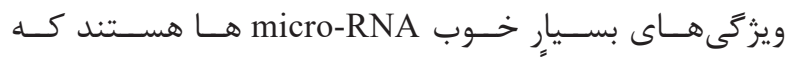

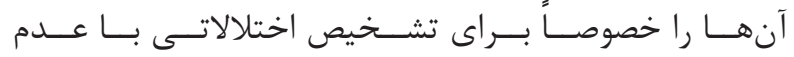

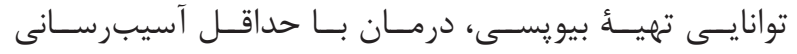

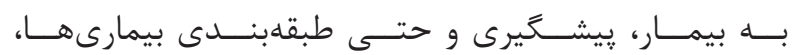

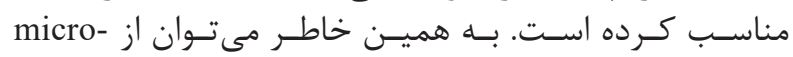

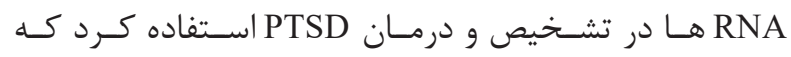

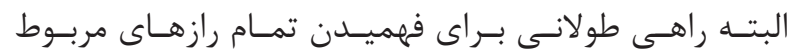

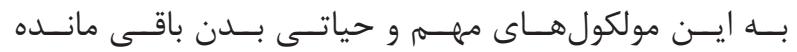

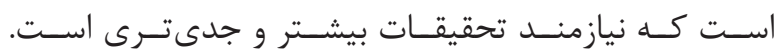

1. Balakathiresan NS, Chandran R, Bhomia M, Jia M, Li H, Maheshwari RK. Serum and amygdala microRNA signatures of posttraumatic stress: fear correlation and biomarker potential. Journal of psychiatric research. 2014; 57: 65-73.

2. Sones HM, Thorp SR, Raskind M. Prevention of posttraumatic stress disorder. Psychiatric Clinics of North America. 2011; 34(1): 79-94.

3. First MB. DSM-5 handbook of differential diagnosis: American Psychiatric Pub; 2013.

4. Psychosocial I. Health status of Vietnam veterans. JAMA. 1988; 259: 2701-7

5. Childress JE, McDowell EJ, Dalai VVK, Bogale SR, Ramamurthy C, Jawaid A, et al. Hippocampal volumes in patients with chronic combat-related postraumatic stress disorder: a systematic review. J Neuropsychiatry Clin Neurosci. 2013; 25(1): 12-25.

6. Van der Kolk BA, McFarlane AC. Traumatic stress: the effects of overwhelming experience on mind, body, and society: Guilford Press; 2012.

7. Alexander W. Pharmacotherapy for post-traumatic stress disorder in combat veterans: focus on antidepressants and atypical antipsychotic agents. Pharmacy and Therapeutics. 2012; 37(1): 32-8.

8. Berna G, Vaiva G, Ducrocq F, Duhem S, Nandrino J. Categorical and dimensional study of the predictive factors of the development of a psychotrauma in victims of car accidents. J Anxiety Disord. 2012; 26(1): 239-45.

9. Gray MJ, Elhai JD, Frueh BC. Enhancing patient satisfaction and increasing treatment compliance: patient education as a fundamental component of PTSD treatment. Psychiatric Quarterly. 2004; 75(4): 321-32.

10. Kroes MC, Rugg MD, Whalley MG, Brewin CR.

$$
\text { نتيجه تيرى }
$$

مطالعـات بسـيار زيـادى در سـال هاى كذشـــه صـورت گرفتـهـ

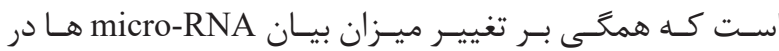

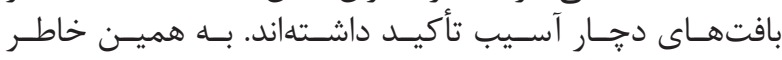

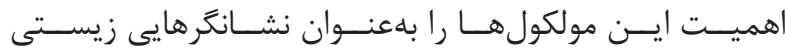

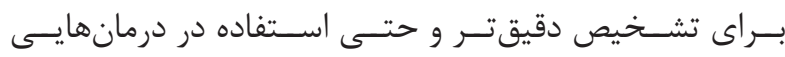

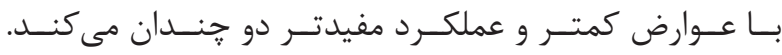

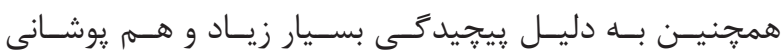

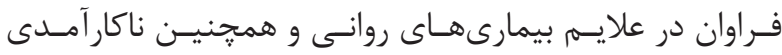

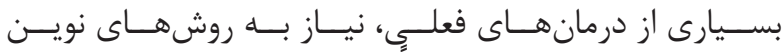

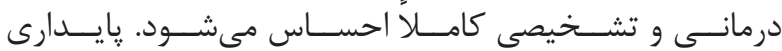

منابع

Structural brain abnormalities common to posttraumatic stress disorder and depression. J Psychiatry Neurosci. 201; 36(4): 256-65.

11. Hawk LW, Dougall AL, Ursano RJ, Baum A. Urinary catecholamines and cortisol in recent-onset posttraumatic stress disorder after motor vehicle accidents. Psychosom Med. 2000; 62(3): 423-34.

12. Solter V, Thaller V, Karlovic D, Crnkovic D. Elevated serum lipids in veterans with combat-related chronic posttraumatic stress disorder. Croat Med J. 2002; 43(6): 685-9.

13. Geracioti Jr TD, Baker DG, Ekhator NN, West $\mathrm{SA}$, Hill KK, Bruce AB, et al. CSF norepinephrine concentrations in posttraumatic stress disorder. Am J Psychiatry. 2001; 158(8): 1227-30.

14. Weathers FW, Keane TM, Davidson JR. Clinicianadministered PTSD scale: a review of the first ten years of research. Depress Anxiety. 2001; 13(3): 132-56.

15. Schmidt U, Kaltwasser SF, Wotjak CT. Biomarkers in posttraumatic stress disorder: overview and implications for future research. Disease Markers. 2013; 35(1): 43-54.

16. Chi Y, Zhou D. MicroRNAs in colorectal carcinomafrom pathogenesis to therapy. J Exp Clin Cancer Res. 2016; 35(1): 43.

17. Kalozoumi G, Yacoub M, Sanoudou D. MicroRNAs in heart failure: small molecules with major impact. Global Cardiology Science and Practice. 2014; 2: 30. doi.org/10.5339/gcsp.2014.30.

18. Baghishani F, Sahab Negah S. The role of neurogenesis in anxiety disorders. Shefaye Khatam. 2017; 5(2): 98-109.

19. Norrholm SD, Jovanovic T, Smith AK, Binder E, 


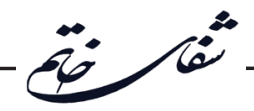

Klengel T, Conneely K, et al. Differential genetic and epigenetic regulation of catechol-O-methyltransferase is associated with impaired fear inhibition in posttraumatic stress disorder. Front Behav Neurosci. 2013; 7: 30. doi: 10.3389/fnbeh.2013.00030.

20. Sahab Negah S, Khaksar Z, Kazemi H, Aligholi H, Safahani M, Modarres Mousavi M, et al. The role of dopamine receptors during brain development. Shefaye Khatam. 2014; 2(3): 65-76

21. Bartel DP. MicroRNAs: genomics, biogenesis, mechanism, and function. Cell. 2004; 116(2): 281-97.

22. Lee RC, Feinbaum RL, Ambros V. The C. Elegans heterochronic gene lin-4 encodes small RNAs with antisense complementarity to lin-14. Cell. 1993; 75(5): 843-54

23. Wahid F, Shehzad A, Khan T, Kim YY. MicroRNAs: synthesis, mechanism, function, and recent clinical trials. Biochimica et Biophysica Acta (BBA)-Molecular Cell Research. 2010; 1803(11): 1231-43.

24. Giridharan V, Thandavarayan R, Fries G, WalssBass C, Barichello T, Justice N, et al. Newer insights into the role of miRNA a tiny genetic tool in psychiatric disorders: focus on post-traumatic stress disorder. Translational Psychiatry. 2016; 6(11): e954.

25. Schöler N, Langer C, Döhner H, Buske C, Kuchenbauer F. Serum microRNAs as a novel class of biomarkers: a comprehensive review of the literature. Experimental Hematology. 2010; 38(12): 1126-30.

26. Fu X, Han Y, Wu Y, Zhu X, Lu X, Mao F, et al. Prognostic role of microRNA-21 in various carcinomas: a systematic review and meta-analysis. European Journal of Clinical Investigation. 2011; 41(11): 1245-53.

27. Sahab Negah S, Mohammad Sadeghi S, Kazemi H, Modarres Mousavi M, Aligholi H. Effect of injured brain extract on proliferation of neural stem cells cultured in 3-dimensional environment. Shefaye Khatam. 2015; 3(1): 49-56.

28. Calin GA, Dumitru CD, Shimizu M, Bichi R, Zupo $\mathrm{S}$, Noch E, et al. Frequent deletions and down-regulation of micro-RNA genes miR15 and miR16 at 13q14 in chronic lymphocytic leukemia. Proceedings of the National Academy of Sciences. 2002; 99(24): 15524-9.

29. Geeleher P, Huang SR, Gamazon ER, Golden A, Seoighe C. The regulatory effect of miRNAs is a heritable genetic trait in humans. BMC Genomics. 2012; 13(1): 383. doi.org/10.1186/1471-2164-13-383.

30. Bartel DP. MicroRNAs: target recognition and regulatory functions. Cell. 2009; 136(2): 215-33.

31. Place RF, Li L-C, Pookot D, Noonan EJ, Dahiya R. MicroRNA-373 induces expression of genes with complementary promoter sequences. Proceedings of the National Academy of Sciences. 2008; 105(5): 1608-13.

32. Dias BG, Goodman JV, Ahluwalia R, Easton AE, Andero R, Ressler KJ. Amygdala-dependent fear memory consolidation via miR-34a and Notch signaling. Neuron. 2014; 83(4): 906-18.

33. Rong H, Liu TB, Yang KJ, Yang HC, Wu DH, Liao CP, et al. MicroRNA-134 plasma levels before and after treatment for bipolar mania. Journal of Psychiatric Research. 2011; 45(1): 92-5.

34. Lai C-Y, Yu S-L, Hsieh MH, Chen C-H, Chen H-Y, Wen $\mathrm{C}-\mathrm{C}$, et al. MicroRNA expression aberration as potential peripheral blood biomarkers for schizophrenia. PloS One. 2011; 6(6): e21635.

35. Sheinerman KS, Tsivinsky VG, Crawford F, Mullan MJ, Abdullah L, Umansky SR. plasma microRNA biomarkers for detection of mild cognitive impairment. Aging (Albany NY). 2012; 4(9): 590- 605.

36. Mohammad Sadeghi S, Sahab Negah S, Khaksar Z, Kazemi H, Aligholi H. Laminin Position as one of the important components of the extracellular matrix in tissue engineering of nervous system. Shefaye Khatam. 2014; 2(1): 69-74.

37. Mahan AL, Ressler KJ. Fear conditioning, synaptic plasticity and the amygdala: implications for posttraumatic stress disorder. Trends in Neurosciences. 2012; 35(1): 24-35.

38. Harrison S, Geppetti P. Substance P. The International Journal of Biochemistry \& Cell Biology. 2001; 33(6): 555-76.

39. Datar P, Srivastava S, Coutinho E, Govil G. Substance P. Structure, function, and therapeutics. Current Topics in Medicinal Chemistry. 2004; 4(1): 75-103.

40. Greco SJ, Rameshwar P. MicroRNAs regulate synthesis of the neurotransmitter substance $\mathrm{P}$ in human mesenchymal stem cell-derived neuronal cells. Proceedings of the National Academy of Sciences. 2007; 104(39): 15484-9.

41. Bankers-Fulbright JL, Kalli KR, McKean DJ. Interleukin-1 signal transduction. Life Sciences. 1996; 59(2): 61-83.

42. Donadeu FX, Schauer S, Sontakke S. Involvement of miRNAs in ovarian follicular and luteal development. Journal of Endocrinology. 2012; 215(3): 323-34 
43. Issler O, Chen A. Determining the role of microRNAs in psychiatric disorders. Nature Reviews Neuroscience. 2015; 16(4): 201-12.

44. Caputo V, Ciolfi A, Macrì S, Pizzuti A. The emerging role of MicroRNA in schizophrenia. CNS \& Neurological Disorders-Drug Targets (Formerly Current Drug Targets-CNS \& Neurological Disorders). 2015; 14(2): 208-21.

45. Suarez-Gomez M, Alejandre-Durán E, Ruiz-Rubio M. MicroRNAs in bipolar disorder: diagnostic and therapeutic applications. Revista De Neurologia. 2011; 53(2): 91-8.

46. Vasu MM, Anitha A, Thanseem I, Suzuki K, Yamada $\mathrm{K}$, Takahashi T, et al. Serum microRNA profiles in children with autism. Molecular Autism. 2014; 5(1): 40. doi.org/10.1186/2040-2392-5-40.

47. Pasand Mozhdeh H, Zeynali B, Aligholi H, Kashani Radgerdi I, Sahab Negah S, Hassanzadeh G. The effect of intracerebroventricular administration of streptozocin on cell proliferation in subventricular zone stem cells in a rat model of alzheimer's disease. Shefaye Khatam. 2015; 3(4): 80-6.

48. Shorter KR, Miller BH. Epigenetic mechanisms in schizophrenia. Progress in Biophysics and Molecular Biology. 2015; 118(1): 1-7.

49. Amunts K, Kedo O, Kindler M, Pieperhoff P, Mohlberg H, Shah N, et al. Cytoarchitectonic mapping of the human amygdala, hippocampal region and entorhinal cortex: intersubject variability and probability maps. Anatomy and Embryology. 2005; 210(5-6): 343-52.

50. Best B. The amygdala and the emotions. Ben Best Science. 2004.

51. Solano-Castiella E, Anwander A, Lohmann G, Weiss M, Docherty C, Geyer S, et al. Diffusion tensor imaging segments the human amygdala in vivo. Neuroimage. 2010; 49(4): 2958-65.

52. Learning P. Why we think plasticity underlying viewpoint Pavlovian fear conditioning occurs in the basolateral amygdala. Neuron. 1999; 23: 229-32.

53. Haramati S, Navon I, Issler O, Ezra-Nevo G, Gil $\mathrm{S}$, Zwang R, et al. MicroRNA as repressors of stressinduced anxiety: the case of amygdalar miR-34. Journal of Neuroscience. 2011; 31(40): 14191-203.

54. Griggs EM, Young EJ, Rumbaugh G, Miller CA. MicroRNA-182 regulates amygdala-dependent memory formation. Journal of Neuroscience. 2013; 33(4): $1734-40$.
55. Van Rooij E, Sutherland LB, Thatcher JE, DiMaio JM, Naseem RH, Marshall WS, et al. Dysregulation of microRNAs after myocardial infarction reveals a role of miR-29 in cardiac fibrosis. Proceedings of the National Academy of Sciences. 2008; 105(35): 13027-32.

56. Mannironi C, Camon J, De Vito F, Biundo A, De Stefano ME, Persiconi I, et al. Acute stress alters amygdala microRNA miR-135a and miR-124 expression: inferences for corticosteroid dependent stress response. PLoS One. 2013; 8(9): e73385.

57. Wingo AP, Almli LM, Stevens JJ, Klengel T, Uddin $\mathrm{M}, \mathrm{Li}$ Y, et al. DICER1 and microRNA regulation in posttraumatic stress disorder with comorbid depression. Nat Commun. 2015; 6: 10106. doi: 10.1038/ncomms10106.

58. Dwivedi Y. Emerging role of microRNAs in major depressive disorder: diagnosis and therapeutic implications. Dialogues Clin Neurosci. 2014; 16(1): 4361.

59. Lin Q, Wei W, Coelho CM, Li X, Baker-Andresen D, Dudley K, et al. The brain-specific microRNA miR$128 \mathrm{~b}$ regulates the formation of fear-extinction memory. Nature Neuroscience. 2011; 14(9): 1115-7.

60. Schmidt U, Herrmann L, Hagl K, Novak B, Huber $\mathrm{C}$, Holsboer F, et al. Therapeutic action of fluoxetine is associated with a reduction in prefrontal cortical miR-1971 expression levels in a mouse model of posttraumatic stress disorder. Front Psychiatry. 2013; 4: 66. doi: 10.3389/fpsyt.2013.00066.

61. Sahab Negah S, Khaksar Z, Mohammad Sadeghi S, Erfanimajd N, Modarres Mousavi M, Aligholi H, et al. Effect of nettle root extract on histometrical parameters of cerebral and cerebellar cortices in rat following administration of testosterone. Shefaye Khatam. 2015; 3(1): 71-8.

62. Meerson A, Cacheaux L, Goosens KA, Sapolsky RM, Soreq H, Kaufer D. Changes in brain MicroRNAs contribute to cholinergic stress reactions. Journal of Molecular Neuroscience. 2010; 40(1-2): 47-55.

63. Schmidt U, Keck ME, Buell DR. miRNAs and other non-coding RNAs in posttraumatic stress disorder: a systematic review of clinical and animal studies. Journal of Psychiatric Research. 2015; 65: 1-8.

64. Restivo L, Vetere G, Bontempi B, Ammassari-Teule $\mathrm{M}$. The formation of recent and remote memory is associated with time-dependent formation of dendritic spines in the hippocampus and anterior cingulate cortex. Journal of Neuroscience. 2009; 29(25): 8206-14.

65. Vetere G, Barbato C, Pezzola S, Frisone P, Aceti 
M, Ciotti M, et al. Selective inhibition of miR-92 in hippocampal neurons alters contextual fear memory. Hippocampus. 2014; 24(12): 1458-6.

66. Sullivan GM, Neria Y. Pharmacotherapy of PTSD: current status and controversies. Psychiatr Ann. 2009; 39(6): 342 .

67. Sahab Negah S, Eshaghabadi A, Mohammadzadeh E. The neuroprotective role of progesterone in traumatic brain injury; reduction of inflammatory cytokines. Shefaye Khatam. 2015; 3(4): 139-50.

68. Bocchio-Chiavetto L, Maffioletti E, Bettinsoli P, GiovanniniC,BignottiS, TarditoD, etal.BloodmicroRNA changes in depressed patients during antidepressant treatment. European Neuropsychopharmacology. 2013; 23(7): 602-11.

69. Camkurt MA, Acar Ş, Coşkun S, Güneş M, Güneş S, Yilmaz MF, et al. Comparison of plasma MicroRNA levels in drug naive, first episode depressed patients and healthy controls. J Psychiatr Res. 2015; 69: 67-71

70. Morey RA, Gold AL, LaBar KS, Beall SK, Brown VM, Haswell CC, et al. Amygdala volume changes in posttraumatic stress disorder in a large case-controlled veterans group. Arch Gen Psychiatry. 2012; 69(11): 1169-78.
71. Zhou J, Nagarkatti P, Zhong Y, Ginsberg JP, Singh NP, Zhang J, et al. Dysregulation in microRNA expression is associated with alterations in immune functions in combat veterans with post-traumatic stress disorder. PLoS One. 2014; 9(4): e940.

72. Issler O, Haramati S, Paul ED, Maeno H, Navon I, Zwang R, et al. MicroRNA 135 is essential for chronic stress resiliency, antidepressant efficacy, and intact serotonergic activity. Neuron. 2014; 83(2): 344-60.

73. Sequeira A, Mamdani F, Ernst C, Vawter MP, Bunney WE, Lebel V, et al. Global brain gene expression analysis links glutamatergic and GABAergic alterations to suicide and major depression. PloS One. 2009; 4(8): e6585.

74. Boyce-Rustay JM, Holmes A. Genetic inactivation of the NMDA receptor NR2A subunit has anxiolytic-and antidepressant-like effects in mice. Neuropsychopharmacology. 2006; 31(11): 2405

75. Liu X, Zhang L, Cheng K, Wang X, Ren G, Xie P. Identification of suitable plasma-based reference genes for miRNAome analysis of major depressive disorder. Journal of Affective Disorders. 2014; 163: 133-9.

76. Dwivedi Y. Emerging role of microRNAs in major depressive disorder: diagnosis and therapeutic implications. Dialogues in Clinical Neuroscience. 2014; 16(1): 43. 